\title{
Inhibitory effects of hyperoside and quercitrin from Zanthoxylum bungeanum Maxim. leaf on 2-amino-1-methyl-6-phenylimidazo [4,5-b] pyridine formation by trapping phenylacetaldehyde
}

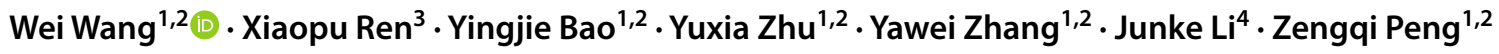

Received: 11 October 2020 / Revised: 15 December 2020 / Accepted: 20 December 2020 / Published online: 12 October 2021

(c) The Author(s) 2021

\begin{abstract}
2-amino-1-methyl-6-phenylimidazole[4,5-b]pyridine (PhIP) is one of the most abundant Heterocyclic amines (HAs) in meat products. Zanthoxylum bungeanum Maxim. leaf (ZML) extract has been shown to be rich in polyphenols, which are gaining increasing interest as efficient tools for inhibiting the formation of HAs. In the present work, the effects of ZML extract, major polyphenols, chlorogenic acid, hyperoside and quercitrin on the formation of PhIP in both roast beef patties and chemical model systems were investigated. UPLC-MS showed that ZML extract and those three polyphenols effectively inhibited PhIP formation. Additionally, GC-MS analysis showed that those three polyphenols significantly reduced the content of phenylacetaldehyde in the model systems, a key intermediate involved in PhIP formation. The subsequent UPLC-MS and TOF-MS/MS analysis found that hyperoside and quercitrin reacted with phenyacetaldehyde to form those four adducts, 8-C-(E-Phenylethenyl)hyperoside, 6-C-(E-Phenylethenyl)hyperoside, 8-C-(E-Phenylethenyl)quercitrin and 6-C-(E-Phenylethenyl)quercitrin, respectively. The results revealed that hyperoside and quercitrin could trap phenylacetaldehyde to form adducts, thereby, retarding the reaction of phenylacetaldehyde and creatinine, blocking the generation of PhIP.
\end{abstract}

Keywords 2-Amino-1-methyl-6-phenylimidazo[4,5-b]pyridine (PhIP) $\cdot$ Polyphenols $\cdot$ Phenylacetaldehyde $\cdot$ Polyphenolphenylacetaldehyde adducts $\cdot$ Model systems $\cdot$ Beef patties

\section{Introduction}

Heterocyclic amines (HAs) are mutagenic and carcinogenic compounds which are formed from sugars, creatine, creatinine, and free amino acids during thermal processing of muscle foods [1, 2]. According to previous studies, HAs may

Junke Li

junjunke@163.com

$\triangle$ Zengqi Peng

zqpeng@njau.edu.cn

1 Jiangsu Collaborative Innovation Center of Meat Production and Processing, Quality and Safety Control, Nanjing Agricultural University, Nanjing 210095, China

2 College of Food Science and Technology, Nanjing Agricultural University, Nanjing 210095, China

3 College of Life Science, Tarim University, Alar 843300, China

4 College of Food Engineering, Ludong University, Yantai 264025, China increase the risk of cancers in different tissues, especially the esophagus, stomach, colon and pancreas [3, 4]. As one of the most abundant HAs in cooked foods [5], 2-amino-1-methyl6-phenylimidazo $[4,5-b]$ pyridine (PhIP) is considered a possible human carcinogen (class 2B) by the International Agency for Research on Cancer [6], induced tumors in non-human primates and increased the risk of second-generation breast cancer [7]. In view of its potent carcinogenic activity and relatively abundant content presence of cooked foods, it is a highpriority to seek strategies to inhibit its formation. Strategies to reduce the PhIP content mainly include two aspects, evaluating the effect of process parameters on PhIP formation and adding plant extracts or natural antioxidants to identify possible inhibitors [8-10]. Mechanistic studies have shown that some flavonoids could reduce the production of $\mathrm{PhIP}$ by reacting with phenylacetaldehyde, an important intermediate involved in PhIP formation, thereby preventing it from entering the pathway of reacting with creatinine to generate $\mathrm{PhIP}[11,12]$.

Zanthoxylum bungeanum Maxim. is a native plant from the Eastern China, which is applied to give special flavor to meat as a traditional spice. As a by-product of Zanthoxylum 
bungeanum Maxim., Zanthoxylum bungeanum Maxim. leaf (ZML) has a unique flavor and rich nutrition. It is also used as a medicine for the treatment of stomach ache, tooth ache and other diseases due to its alkaloids, polyphenols, and so on [13]. However, ZML is usually discarded directly, which may give rise to high risk to the environment and result in a waste of resources and economy. Therefore, to reduce the resources waste and environmental pressure, it is necessary to optimize the utilization of ZML. Fortunately, a variety of polyphenols, including phenolic acids and flavonoids, have been identified in ZML extract, of which, chlorogenic acid is the most abundant phenolic acid, hyperoside and quercitrin are the two major flavonoids [14]. Polyphenols have gained wide attention for the potent antioxidant and broad-spectrum antibacterial activities [15], especially their excellent performance in inhibiting HAs formation [12]. The inhibitory effects of chlorogenic acid, ferulic acid, $p$-coumaric acid and protocatechuic acid on HAs formation in chemical model systems were investigated by Zeng et al. [16]. Rosmarinic acid was able to reduce the content of PhIP in beef patties [17]. Although the effects of these phenolic acids on HAs formation have been evaluated, no report was found on the mechanism behind the inhibitory effects of chlorogenic acid, especially with reference to the natural ratios of ZML. As for flavonoids, naringenin, quercetin and EGCG have been proven to inhibit PhIP formation via trapping of phenylacetaldehyde to form adducts [12, $18,19]$. The introduction of 3-O-glucoside of quercetin leads to the formation of quercitrin, which shows similar structural characteristics to hyperoside. However, it is still unknown that whether all of flavonoids possess the similar inhibition and mechanisms due to the differences in the substituent groups. Thus it is necessary to examine the inhibitory effects of ZML extract and its components on PhIP formation.

In the present work, the effects of ZML extract, including chlorogenic acid, hyperoside and quercitrin, on PhIP formation in both roast beef patties and chemical model systems were investigated. Furthermore, the potential mechanisms behind the inhibitory effects were explored. These would not only develop promising strategies to inhibit PhIP formation, but also provide valuable information to enhance food safety.

\section{Materials and methods}

\section{Reagents and materials}

Phenylalanine, glucose, creatinine, di(ethylene) glycol, phenylacetaldehyde, chlorogenic acid, hyperoside and quercitrin were obtained from Sigma-Aldrich (Shanghai, China). The PhIP standard was purchased from Toronto Research Chemicals (Downsview, Ontario, Canada). Oasis MCX (60 mg, $3 \mathrm{~mL}$ ) cartridges were supplied by Waters (Milford, MA,
USA). HPLC or analytical grade chemicals and solvents were used in this study.

\section{Preparation of ZML extract}

The ZML extract was obtained according to the method reported by Li et al. [14]. The ZML powder was mixed with $65 \%(\mathrm{v} / \mathrm{v})$ ethanol solution and extracted using a microwave for $30 \mathrm{~min}$. The ethanol extract was filtered and concentrated and then was freeze-dried to obtain the ZML extract.

\section{The levels of the polyphenols in beef patties and model systems}

The concentrations of chlorogenic acid, hyperoside and quercitrin were $2.67,8.25$ and $11.62 \mathrm{~g} / 100 \mathrm{~g}$ of ZML extract, respectively [14]. The addition of ZML extract in the present work referred to the data of $\mathrm{Li}$, with the three levels of 0.015 , 0.030 and $0.045 \%(\mathrm{w} / \mathrm{w})$. The amounts of level 1 for chlorogenic acid, hyperoside and quercitrin were equivalent to the concentrations of the three polyphenols in the $0.015 \%$ ZML extract, respectively. The levels of reactants in the model systems were 35 times of the content of phenylalanine and creatinine in raw beef $(951$ and $710 \mathrm{mg} / \mathrm{kg}$, respectively; the creatine content of the raw beef was $4250 \mathrm{mg} / \mathrm{kg}$ ), and the concentrations of the polyphenols were also 35 times of that in the patties. All the levels are listed in Table 1.

\section{Preparation of beef patties}

The ground beef was mixed with different levels of ZML extract, chlorogenic acid, hyperoside and quercitrin, and then was formed into beef patties. The patties were incubated for $8 \mathrm{~h}$ at $4{ }^{\circ} \mathrm{C}$ and then roasted for $10 \mathrm{~min}$ on each side at $225^{\circ} \mathrm{C}$ in an electric oven (D3-256A, Toshiba, Japan). Next, the patties were cut into small pieces and freeze-dried. All the above experiments were performed in triplicate.

The extraction process of PhIP refers to Zeng et al. [9]. The lyophilized patty samples $(5.0 \mathrm{~g})$ were placed into $30 \mathrm{~mL}$ of $1 \mathrm{M} \mathrm{NaOH}$ and homogenized for $2 \mathrm{~min}$. Thereafter, the homogenates were thoroughly mixed with $13 \mathrm{~g}$ of diatomaceous earth, and then extracted with $40 \mathrm{~mL}$ of ethyl acetate for 30 min twice. The concentrated fluent was transferred to Waters Oasis MCX cartridges, which were then sequentially rinsed with $6 \mathrm{~mL}$ of $\mathrm{HCl}(0.1 \mathrm{M})$, methanol, and a methanolammonia mixture $(19: 1, \mathrm{v} / \mathrm{v})$ and concentrated under nitrogen flushing. The residue was filtered through a $0.22-\mu \mathrm{m}$ syringe filter and dissolved in $250 \mu \mathrm{L}$ of methanol before UPLC-MS analysis. 
Table 1 Levels of the three polyphenols in roast beef patties and the model systems

\begin{tabular}{lllllll}
\hline Groups & \multicolumn{2}{l}{ Beef patties } & \multicolumn{3}{l}{ Model systems } \\
\hline & $\begin{array}{l}\text { Level }_{\mathrm{p}} 1 \\
(\mu \mathrm{g} / \mathrm{g})\end{array}$ & $\begin{array}{l}\text { Level }_{\mathrm{p}} 2 \\
(\mu \mathrm{g} / \mathrm{g})\end{array}$ & $\begin{array}{l}\text { Level }_{\mathrm{p}} 3 \\
(\mu \mathrm{g} / \mathrm{g})\end{array}$ & Level $_{\mathrm{m}}$ 1 $(\mathrm{mM})$ & Level $_{\mathrm{m}} 2(\mathrm{mM})$ & Level $_{\mathrm{m}} 3(\mathrm{mM})$ \\
\hline Chlorogenic acid & 5 & 10 & 15 & 0.15 & 0.30 & 0.45 \\
Hyperoside & 15 & 30 & 45 & 0.30 & 0.60 & 0.90 \\
Quercitrin & 20 & 40 & 60 & 0.40 & 0.80 & 1.20 \\
\hline
\end{tabular}

Level $_{\mathrm{p}}$ represents the level of each additive in roast beef patties. Level ${ }_{\mathrm{m}}$ represents the additive level in the model systems. The numbers (1,2 and 3 ) represent the different additive levels

\section{Effects of the polyphenols on PhIP formation in the model systems}

Different levels of chlorogenic acid, hyperoside and quercitrin were added to the reaction vials respectively. The control model system comprised $2 \mathrm{mmol}$ phenylalanine, $2 \mathrm{mmol}$ creatinine and $1 \mathrm{mmol}$ glucose. The reaction medium was $10 \mathrm{~mL}$ di(ethylene) glycol containing $14 \%$ water. The mixtures were heated at $200{ }^{\circ} \mathrm{C}$ in closed test tubes for $1 \mathrm{~h}$. After heating, the tubes were immediately cooled down in an ice bath to terminate the reaction. The samples were subsequently prepared for UPLC-MS analysis. The experiments were performed in triplicate.

\section{UPLC-MS analysis of PhIP}

To identify and quantify PhIP, we used an Acquity UPLC BEH C18 column $(1.7 \mu \mathrm{m} ; 2.1 \mathrm{~mm} \times 100 \mathrm{~mm}$ I.D.; Waters) at $35^{\circ} \mathrm{C} .10 \mathrm{mmol} / \mathrm{L}$ of ammonium acetate $(\mathrm{pH} 6.8)$ and acetonitrile were mobile phases $\mathrm{A}$ and $\mathrm{B}$, respectively. The solvent composition was 0-0.1 min, $90 \% \mathrm{~A}$; 0.1-18 min, $10-30 \% \mathrm{~B}$; 18-20 min, 30-100\% B; 20-20.1 min, 100-10\% B. The total flow rate was $0.3 \mu \mathrm{L} / \mathrm{min}$, and the injection volume was $2 \mu \mathrm{L}$. The MS conditions were as follows: the positive ion mode; capillary voltage, $3.5 \mathrm{kV}$; ion source temperature, $120^{\circ} \mathrm{C}$; desolvation temperature, $400^{\circ} \mathrm{C}$. Data acquisition and processing were performed using Masslynx 4.1 (Waters, Milford, MA, USA).

\section{Evaluation of phenylacetaldehyde-scavenging capabilities in the model systems by GC-MS}

The model system to examine the capabilities of phenylacetaldehyde-scavenging was based on previous studies [12]. Ten milliliters of the reaction mixture was extracted with $20 \mathrm{~mL}$ of ethyl acetate. After thoroughly mixing, anhydrous sodium sulfate was added for drying. The mixtures were filtered through a $0.22-\mu \mathrm{m}$ syringe filter immediately before GC-MS analysis. The samples were analyzed by Agilent gas chromatography (Agilent 7890) coupled with an Agilent mass spectrometer (5975 MSD) with a triple axis. The separation was carried out using an HP-5 capillary column. Analyses were performed using the following parameters: inlet temperature, $200{ }^{\circ} \mathrm{C}$; column flow, $1 \mathrm{~mL} / \mathrm{min}(\mathrm{He})$; temperature program, $40{ }^{\circ} \mathrm{C}$ for $4 \mathrm{~min}, 5^{\circ} \mathrm{C} / \mathrm{min}$ up to $230{ }^{\circ} \mathrm{C}$ and hold for $5 \mathrm{~min}$.

\section{Direct reaction between polyphenol and phenylacetaldehyde in the di(ethylene) glycol model systems}

This method was primarily from the study of Zhu et al. [12]. First, the polyphenols and phenylacetaldehyde were added to $10 \mathrm{~mL}$ solution of di(ethylene) glycol containing 14\% MilliQ water. Second, the mixtures were heated at $200{ }^{\circ} \mathrm{C}$ for $1 \mathrm{~h}$. Next, the reaction solution was cooled down in an ice bath. After cooling, the extraction solvent system was prepared as follows: sample:water:ethyl acetate: hexane $=1: 1: 2: 1(\mathrm{v} / \mathrm{v})$. The solvent mixture was then vortexed for $3 \mathrm{~min}$ and centrifuged at $8000 \mathrm{~g}$ for $10 \mathrm{~min}$. Finally, the supernatant was evaporated and the resulting extract was redissolved in methanol. The adducts were detected using a Waters UPLC-Q-TOF system.

\section{Extraction and detection of adducts in roast beef patties}

Ten grams of the patty powder was added to $50 \mathrm{ml}$ of MilliQ water and homogenized for $2 \mathrm{~min}$ and then extracted twice with $100 \mathrm{ml}$ of ethyl acetate. The extract was centrifuged at $8000 \mathrm{~g}$ for $10 \mathrm{~min}$, and the supernatant was collected and 
rotary evaporated. Finally, the mixtures were dissolved in methanol and filtered before UPLC-MS analysis. The experiments were performed in triplicate.

\section{UPLC-MS analysis of polyphenol-phenylacetaldehyde adducts}

The adducts were identified by an UPLC-MS system (G2XS Q-TOF Waters) equipped with a Waters Acquity UPLC. The separation was performed using an Acquity UPLC BEH C18 column $(100 \mathrm{~mm} \times 2.1 \mathrm{~mm}, 1.7 \mu \mathrm{m}) .0 .1 \%$ formic acid in water (buffer A) and methanol (buffer B) were mobile phases. The elution gradient was $5 \%$ buffer B for $0.5 \mathrm{~min}$, 5-95\% buffer B for $11 \mathrm{~min}$, and $95 \%$ buffer B for 2 min at a flow rate of $0.4 \mathrm{~mL} / \mathrm{min}$. An electrospray source in the positive ion mode with the MSe acquisition mode were used for Mass spectrometry analysis. The MS conditions were as follows: capillary voltage $2.5 \mathrm{kV}$; scan range $50-1200 \mathrm{~m} / \mathrm{z}$; collision energy $40 \mathrm{eV}$; source temperature $120^{\circ} \mathrm{C}$; desolvation gas temperature $400{ }^{\circ} \mathrm{C}$.

\section{Statistical analysis}

All the experiments were conducted in triplicate. The data were expressed as the mean \pm standard deviation. Data analysis was performed using the IBM SPSS Statistics program ver. 19 (Chicago, IL, USA), and differential significance analysis was performed using Duncan's multiple range test. $P<0.05$ indicated a statistically significant difference.

\section{Results and discussion}

\section{Effects of ZML extract on PhIP formation in the patties}

UPLC-MS analysis was used to detect the content of PhIP in the patties with and without ZML extract. The correlation coefficient $\left(R^{2}\right)$ for PhIP standard curve was 0.9982 . The limit of detection (LOD) and limit of quantification (LOQ) adopt the signal-to-noise ratio $(\mathrm{S} / \mathrm{N})$ of 3 and 10 , respectively. The LOD for PhIP was $0.080 \mathrm{ng} / \mathrm{g}$ and the LOQ was $0.112 \mathrm{ng} / \mathrm{g}$. These values were consistent with the previous studies [20]. As shown in Fig. 1, the contents of PhIP in all treatments were significantly lower than those of the control $(p<0.05)$ and decreased with the increasing level of ZML extract. ZML extract significantly reduced the formation of PhIP by $40-78.02 \%$. Similar results with a reduction of approx. 90\% for PhIP using grape seed and rosemary extract in marinades were shown in a study of Gibis et al. [10]. These results suggested that the ZML extract was a potent inhibitor against

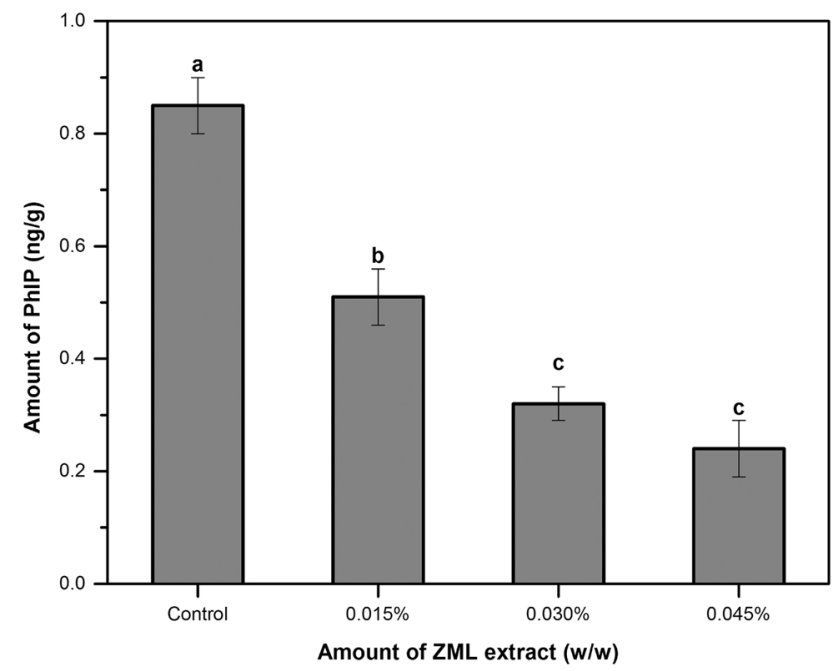

Fig. 1 Inhibitory effects of different concentrations of ZML extract on the formation of PhIP in roast beef patties $(n=3)$. Different letters $(\mathrm{a}-\mathrm{c})$ above the error bars indicate significant differences $(p<0.05)$

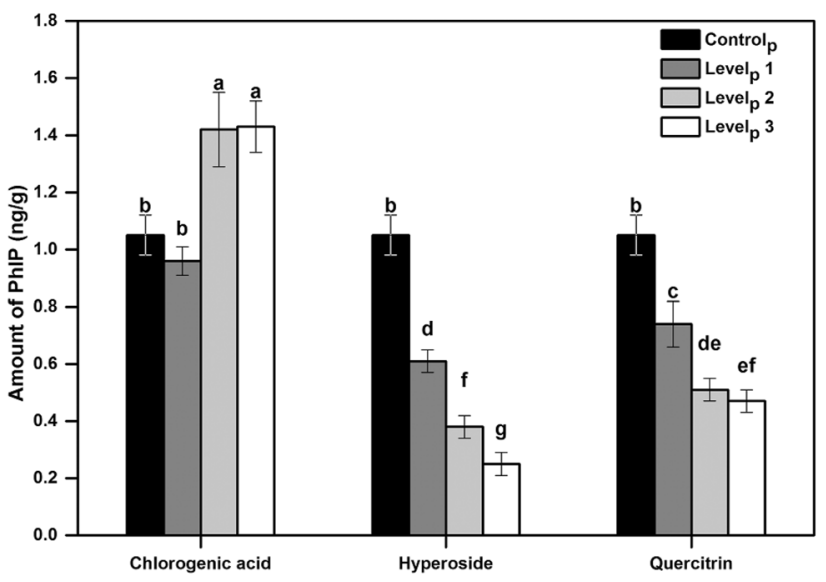

Fig. 2 Inhibitory effects of different levels of chlorogenic acid, hyperoside and quercitrin on the formation of PhIP in roast beef patties $(n=3)$. Level ${ }_{\mathrm{p}}$ represents the additive levels in the patties. Different letters (a-g) above the error bars indicate significant differences $(p<0.05)$

PhIP formation. Numerous studies have also found that natural extracts effectively inhibit HAs formation, which may be related to the polyphenols in the extracts $[21,22]$.

\section{Effects of the polyphenols on PhIP formation in the patties and model systems}

To identify the active ingredients of ZML extract that inhibited PhIP formation, chlorogenic acid, hyperoside and quercitrin were further evaluated. The results showed that 
each polyphenol influenced $\mathrm{PhIP}$ formation in the patties (Fig. 2). Hyperoside and quercitrin significantly $(p<0.05)$ inhibited PhIP generation. The highest level of hyperoside reduced PhIP formation by $76.19 \%$. Zhu et al. [12] tested eight flavonoids for their effects on HAs formation, among which phlorizin and EGCG exhibited the strongest inhibition effects on PhIP formation, with inhibition rates of up to $78.56 \%$ and $77.45 \%$, respectively. A similar inhibitory effect was seen in quercitrin, suggesting that quercitrin is also an efficient inhibitor on PhIP formation. Contrary to hyperoside and quercitrin, chlorogenic acid at higher levels

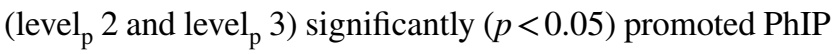
formation, which was capable of increasing the amount of PhIP by $36.19 \%$.

Having found that the polyphenols significantly inhibited PhIP formation in roast beef patties, their inhibitory effects were further evaluated in chemical model systems (Fig. 3). As expected, hyperoside and quercitrin effectively inhibited PhIP generation $(p<0.05)$. The inhibition rates of PhIP ranged from $49.23 \%$ to $63.86 \%$. Different from the patties, chlorogenic acid inhibited PhIP formation in the model systems, with the maximum inhibition rate of $61.77 \%$. Similar observation with different polyphenols, which suppressed the formation of PhIP in both the meat and model systems, has been also reported. Cheng et al. [23] reported that naringenin significantly reduced the amount of PhIP in the model systems and beef patties, with the inhibitory rate of nearly $60 \%$ and $74 \%$, respectively. Zhou et al. [24] showed a significant decrease on PhIP formation by the addition of dihydromyricetin to both the fried beef patties and the model systems, with the maximum inhibitory rate up to $80.1 \%$ and $75.7 \%$, respectively.

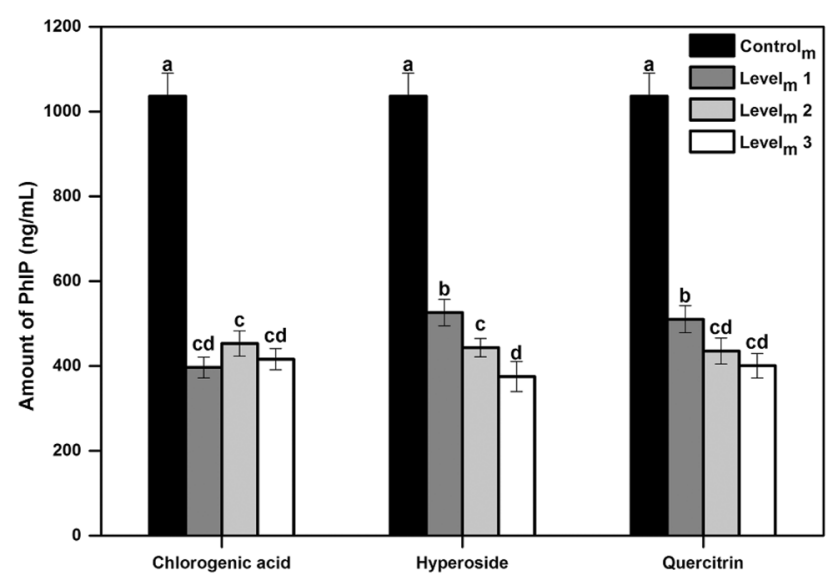

Fig. 3 Inhibitory effects of different levels of chlorogenic acid, hyperoside and quercitrin on the formation of PhIP in model systems

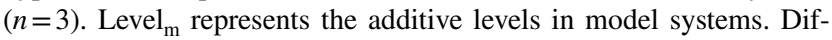
ferent letters $(\mathrm{a}-\mathrm{d})$ above the error bars indicate significant differences $(p<0.05)$
Comparing Figs. 2 and 3, it was found that chlorogenic acid showed opposite effects on PhIP formation in the two systems, which were consistent with other investigations [17, 23]. Possible explanations are that the formation mechanisms of PhIP may differ between the model systems and meat, and the difference in the composition of the two systems may have a larger effect on the role of the added polyphenols on PhIP formation [25]. Moreover, some reactive groups from phenolic acids, such as the carboxyl group, interact with the amino group of phenylalanine, possibly affecting PhIP formation [26].

Among the three polyphenols, hyperoside and quercitrin showed relatively strong inhibitory effects on PhIP formation in both systems. These results suggested that hyperoside and quercitrin may be promising candidates for PhIP-inhibition agents. Different structures were in charge of different functions. The similarity in their structures lies in the presence of hydroxyl groups at the 3'- and 4'-position of the B-ring, which increases the inhibition on PhIP formation to a certain extent. Despite the above mentioned similarities, important differences among them also could be recognized. On the one hand, the presence of a pair of meta hydroxyl groups on the A-ring in hyperoside and quercitrin but not in chlorogenic acid has a predominant contribution to their inhibitory abilities. On the other hand, the existence of a double bond between the 2- and 3-positions of the C-ring of hyperoside and quercitrin also improves their inhibitory abilities. In summary, the above differences may explain that the ability of chlorogenic acid to inhibit PhIP formation in the patties is relatively weaker than that of the other two polyphenols.

\section{Phenylacetaldehyde-scavenging capabilities of polyphenols in the model systems}

Previous studies have shown that phenylacetaldehyde is a key intermediate of PhIP, playing an important role in the formation of PhIP [27]. Numerous studies revealed that flavonoids cause significant inhibition effects on PhIP formation by directly reacting with phenylacetaldehyde [11, 12 , 19]. Thus, it is critical to assess the phenylacetaldehydescavenging capabilities of the polyphenols. In the present work, the scavenging abilities of phenylacetaldehyde by chlorogenic acid, hyperoside and quercitrin were determined by GC-MS. The results (Fig. 4a) showed that all the polyphenols significantly reduced the amount of phenylacetaldehyde in the model systems $(p<0.05)$. At the highest level, chlorogenic acid, hyperoside and quercitrin showed the strongest abilities to trap phenylacetaldehyde, with trapping ratios of up to $54.65,62.52$ and $67.37 \%$, respectively. The amount of phenylacetaldehyde treated with hyperoside at level $\mathrm{l}_{\mathrm{m}} 1$ was significantly lower than those of chlorogenic acid and quercitrin at level $\mathrm{m}_{\mathrm{m}} 1(p<0.05)$, suggesting that 


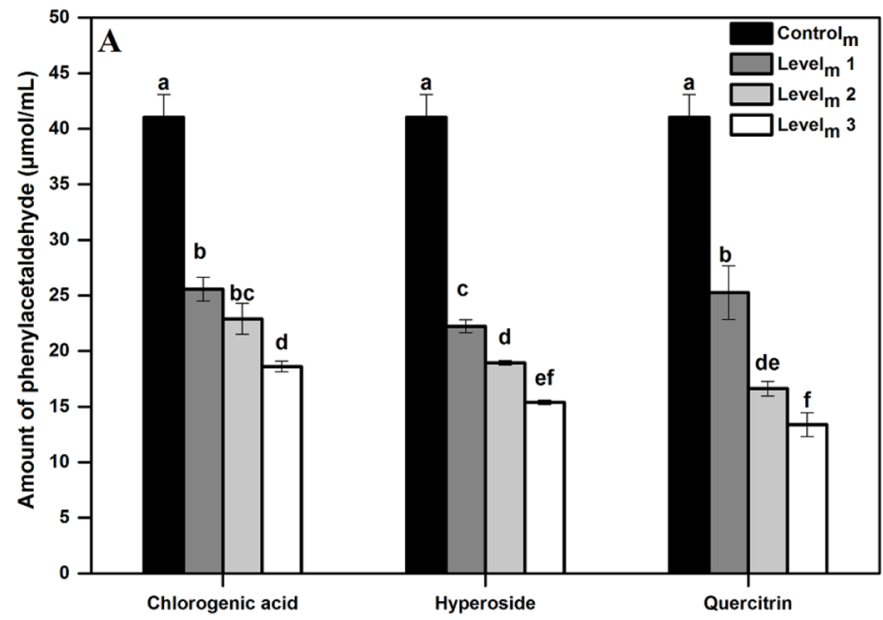

Fig. 4 Effects of different concentrations of chlorogenic acid, hyperoside and quercitrin (a) on the amount of phenylacetaldehyde in the model systems $(n=3)$ and carbonyl-scavenging region of polyphenols

hyperoside exhibited stronger capabilities to trap phenylacetaldehyde. By contrast, chlorogenic acid showed relatively weak phenylacetaldehyde-scavenging capabilities.

Zamora et al. [28] suggested that the carbonyl-scavenging activity requires the presence of phenolic carbons with a high electronic density, such as those in luteolin, apigenin, chrysin and baicalein. This result indicated that hyperoside and quercitrin also contain active carbonyl-scavenging regions, which was consistent with the strong trapping capabilities of phenylacetaldehyde of these two polyphenols. However, chlorogenic acid, with smaller active carbonylscavenging regions, would exhibit relatively weak phenylacetaldehyde-scavenging capabilities.

\section{Relationships \\ between the phenylacetaldehyde-trapping and PhIP-inhibiting abilities of the polyphenols}

The relationships between the effects of the polyphenols on PhIP formation and their phenylacetaldehyde-scavenging capabilities in di(ethylene) glycol reaction solution in both the patties and model systems were analyzed using a linear regression model. Good correlations were obtained between the effects of hyperoside $\left(R^{2}=0.8928\right.$ and 0.9113 ; Fig. $\left.5 \mathrm{~b}\right)$ $(p<0.01)$ and quercitrin $\left(R^{2}=0.8699\right.$ and 0.7683 ; Fig. 5 c $)$ $(p<0.01)$ on PhIP formation and their phenylacetaldehydescavenging abilities in both systems. The results indicated that the effect of polyphenols on PhIP formation is closely related to their corresponding phenylacetaldehyde-scavenging abilities. Therefore, the inhibition of PhIP by hyperoside and quercitrin is likely based on their mechanisms

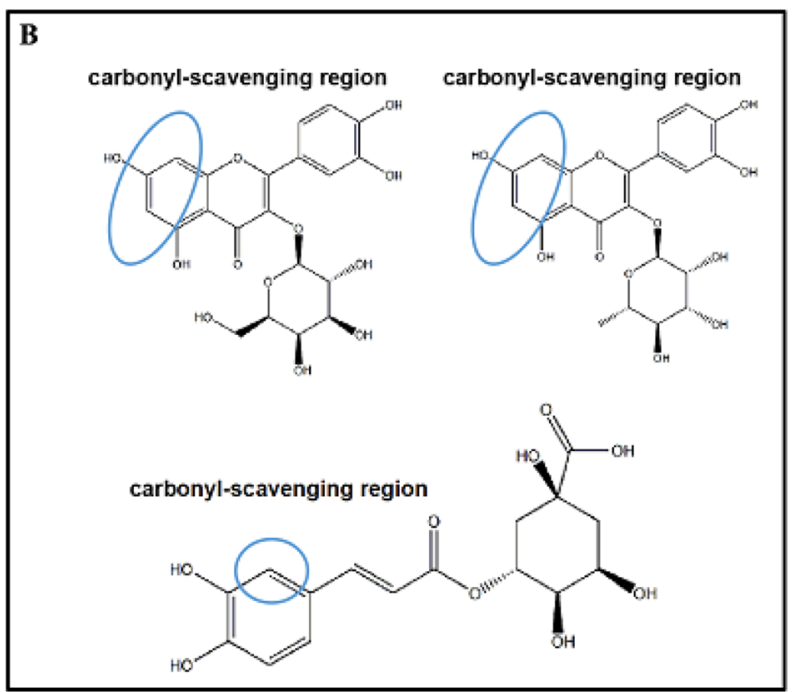

(b). Level $_{\mathrm{m}}$ represents the additive levels in the model systems. Different letters $(a-f)$ above the error bars indicate significant differences $(p<0.05)$

of trappinging phenylacetaldehyde. However, the data of chlorogenic acid showed a poor correlation $\left(R^{2}=0.5002\right.$ and 0.0078; Fig. 5a). Thus, other potential pathways warrant further investigation considering that chlorogenic acid had a strong ability to inhibit PhIP formation in the model systems.

\section{Analysis of polyphenol-phenylacetaldehyde adducts in the patties and model systems}

To gain insight into the mechanisms underlying how the polyphenols scavenge phenylacetaldehyde, UPLC-MS analysis was performed to identify reaction products that may arise from reactions between phenylacetaldehyde and the polyphenols. In the present work, four adducts were identified in the model systems, namely $8-\mathrm{C}-(E$-phenylethenyl $)$ hyperoside $), 6-\mathrm{C}-(E$-phenylethenyl)hyperoside, 8-C-(Ephenylethenyl)quercitrin and 6-C-(E-phenylethenyl)quercitrin (Fig. 6). The total ion chromatogram (TIC) of the reaction mixtures of phenylacetaldehyde and hyperoside is shown in Fig. 6a1. Two extracted molecular ion peaks at $\mathrm{m} / \mathrm{z} 567.15\left[(\mathrm{M}+\mathrm{H})^{+}\right]$in positive ESI-MS were detected, and the corresponding molecular weight was 566 , assignable to a product of hyperoside and phenylacetaldehyde with the elimination of a water molecule. Similarly, for quercitrin (Fig. 6a2), two main molecular ion peaks appeared at $\mathrm{m} / \mathrm{z} 551.16\left[(\mathrm{M}+\mathrm{H})^{+}\right]$in positive ESI-MS with a molecular weight of 550, which agreed with the molecular weight of quercitrin and phenylacetaldehyde after removing one molecule of water. Moreover, the roast beef patties treated 


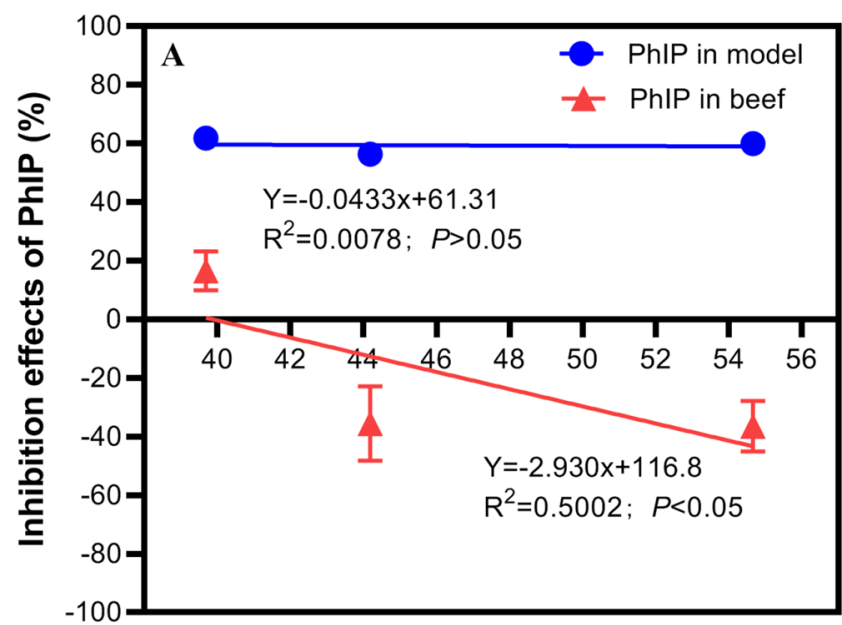

Trapping capabilities of phenylacetaldehyde (\%)

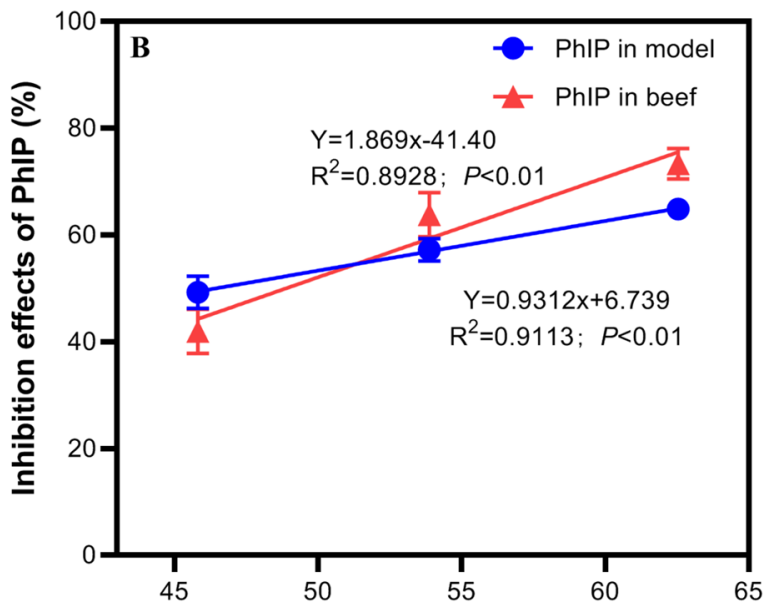

Trapping capabilities of phenylacetaldehyde (\%)

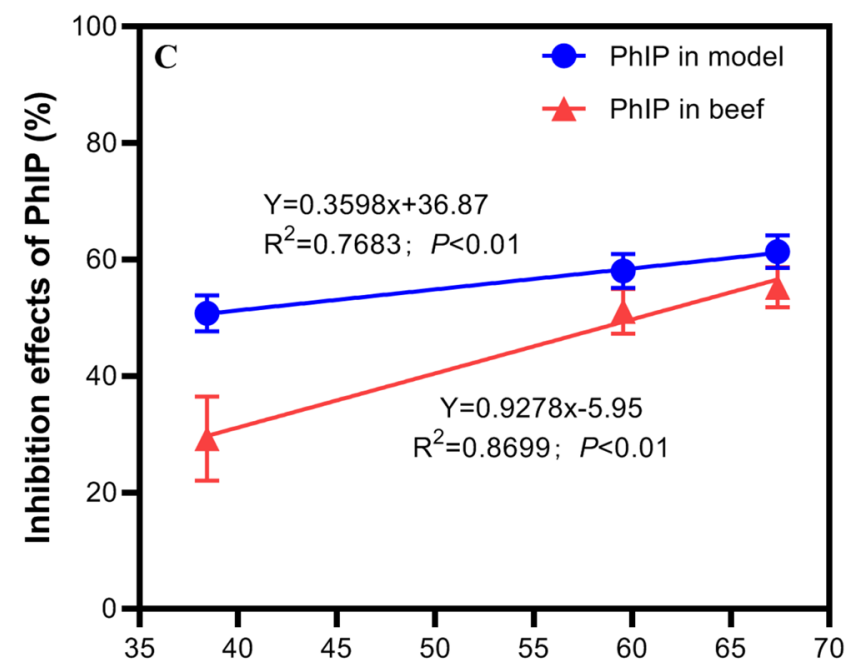

Trapping capabilities of phenylacetaldehyde (\%)

Fig. 5 Correlations of the different levels of chlorogenic acid (a), hyperoside (b) and quercitrin (c) on the phenylacetaldehyde-scavenging rate and the inhibition rates of PhIP formation in the patties and model systems $(n=3)$

with hyperoside and quercitrin also contained the identified adducts (Fig. 6b).

Target adducts formed from hyperoside and quercitrin were further examined by TOF-MS/MS. According to the analysis results of collision-induced dissociation (CID) of hyperoside-phenylacetaldehyde adducts at $\mathrm{m} / \mathrm{z} 567.15$ $\left[(\mathrm{M}+\mathrm{H})^{+}\right]$, two major fragment ions were generated, $\mathrm{m} / \mathrm{z}$ 405 and $\mathrm{m} / \mathrm{z} 255$, respectively. And the CID of the $\mathrm{m} / \mathrm{z}$ $551.16\left[(\mathrm{M}+\mathrm{H})^{+}\right]$ion also produced major fragment ions at $\mathrm{m} / \mathrm{z} 405$ and $\mathrm{m} / \mathrm{z} 255$. The fragment ion of $\mathrm{m} / \mathrm{z} 405$ was mainly due to the loss of the glycosidic bond at 3-position from the $\mathrm{C}$-ring. The appearance of $\mathrm{m} / \mathrm{z} 255$ was assigned to the Retro-Diels-Alder cleavage of the C-ring from the $\mathrm{m} / \mathrm{z} 405$, thus precluding the B-ring and C-ring as the active trapping site for phenylacetaldehyde, and further indicating that the A-ring is the trapping site. Zhu et al. [29] explained that the hydroxyl groups at the meta position act as electron donors and could form electronrich centers at the unsubstituted carbon sites to promote the electrophilic substitution reactions with electrophiles such as reactive carbonyl species. Furthermore, numerous studies $[12,18,19]$ have revealed that some polyphenols scavenge phenylacetaldehyde via electrophilic substitution reactions at C-6 and C-8 of the A-ring. In conclusion, given the unsubstituted carbon sites on the A-ring of hyperoside and quercitrin, we conclude that C-6 and C-8 are active sites for trapping phenylacetaldehyde.

The above results indicated that in the presence of hyperoside and quercitrin, phenylacetaldehyde was trapped by forming adducts, thereby preventing it from entering 

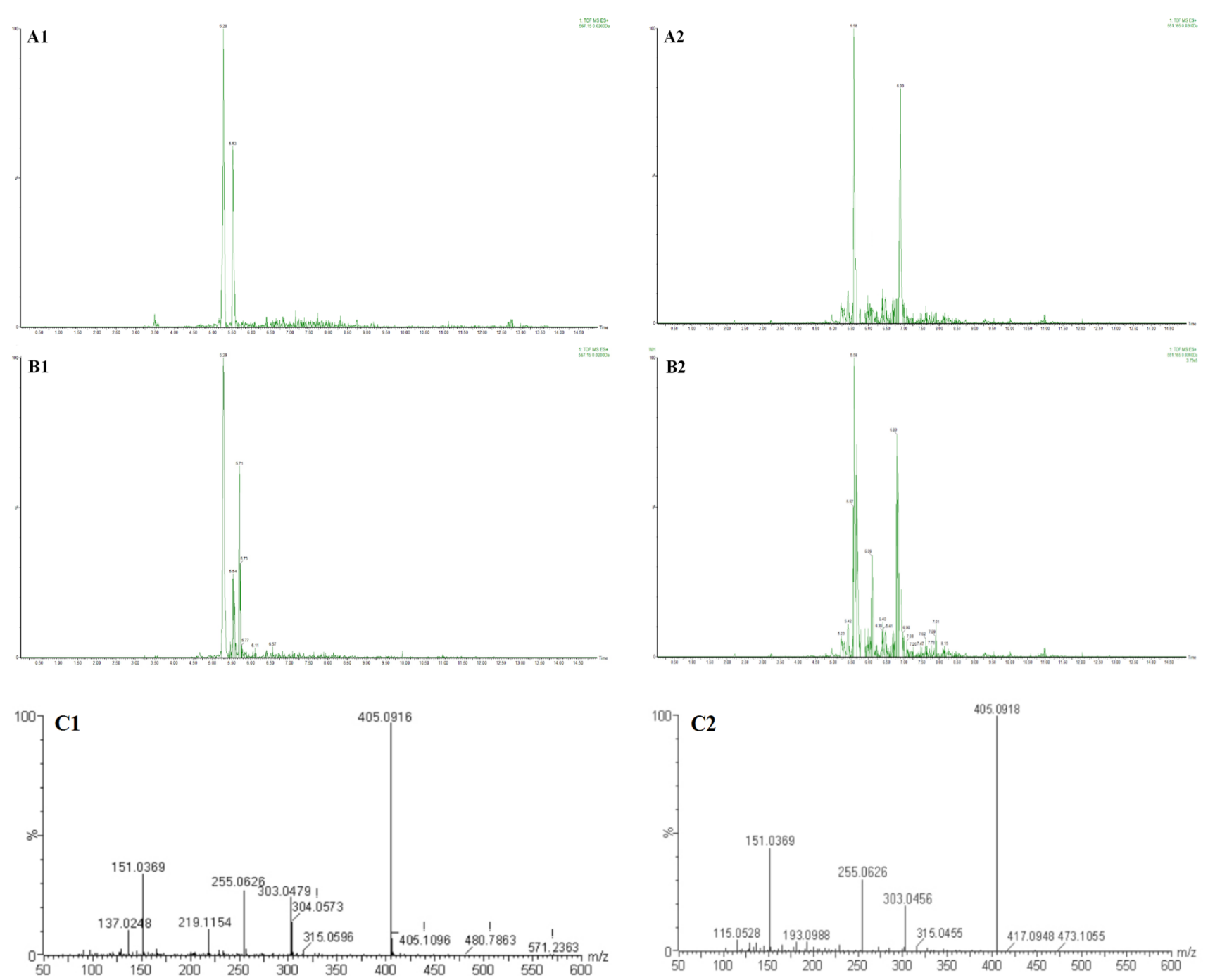

Fig. 6 Polyphenol-phenylacetaldehyde adducts in the chemical reaction systems and roast beef patties. Extracted ion chromatograms of polyphenol-phenylacetaldehyde adducts in the chemical reaction sys-

the pathway leading to the formation of PhIP. Postulated pathways for the inhibitory activity of hyperoside and quercitrin on PhIP formation was exhibited in Fig. 7. However, the mechanism of chlorogenic acid on the inhibition of PhIP formation in the model systems is unknown. Chlorogenic acid significantly inhibited PhIP formation, but did not generate a predicted adduct with phenylacetaldehyde (Supplementary Fig. S1). Zhang et al. [30] reported that caffeic acid releases a $\mathrm{CO}_{2}$ molecule to produce 4-vinylcatechol, which could form adducts with phenylacetaldehyde, thus leading to significant inhibition of PhIP formation. Dihydromyricetin was also demonstrated to be degraded to myricetin during heating and then traps phenylacetaldehyde to form adducts, thereby inhibiting PhIP formation [24]. Therefore, it was speculated that chlorogenic acid may undergo degradation to generate some substances tems (a) and roast beef patties (b), TOF-MS/MS spectrum of polyphenol-phenylacetaldehyde adducts (c)

after heating in the model systems, and these substances may have good ability to inhibit PhIP formation.

Some of these flavonoid-phenylacetaldehyde adducts were reported to exhibit anticancer potential, among which 8-C- and 6-C-(E-phenylethenyl)naringenin showed selective cytotoxicity and anticancer activity against colon cancer cells in a mouse model of human colon cancer [31]. 8-C-(E-phenylethenyl)norartocarpetin significantly induced cancer cell death [32]. Thus it can be hypothesized that polyphenol-phenylacetaldehyde adducts detected in the present work might also exhibit anticancer potential. The above speculations need further identification. 


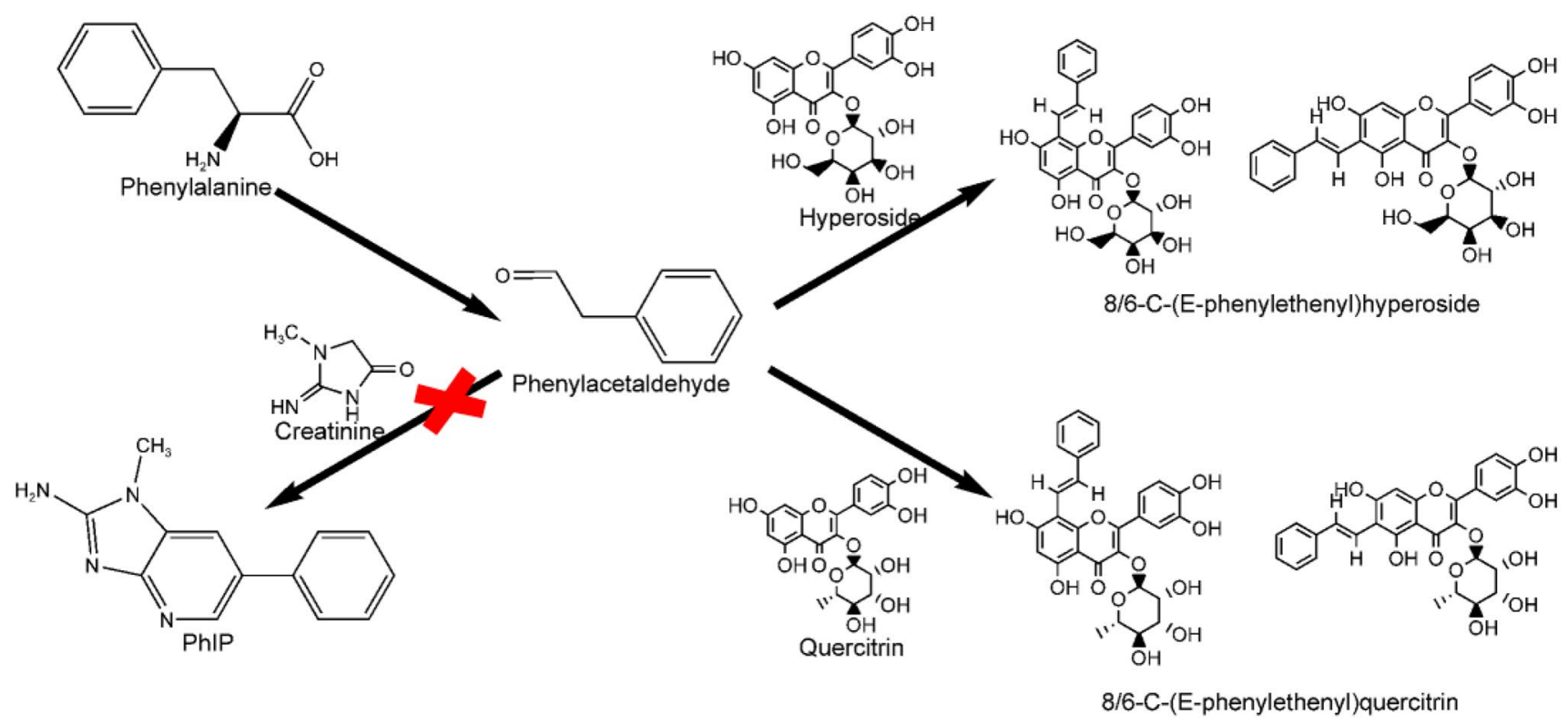

Fig. 7 Postulated pathways for the inhibitory activity of hyperoside and quercitrin on $\mathrm{PhIP}$ formation

\section{Conclusions}

It was concluded that ZML extract, hyperoside and quercitrin significantly inhibit PhIP formation in both beef patties and chemical model systems. Further identification of 8-C-(E-phenylethenyl)hyperoside, 6-C-( $E$-phenylethenyl) hyperoside, 8-C-(E-phenylethenyl)quercitrin and 6-C- $(E-$ phenylethenyl)quercitrin in the two systems confirmed that the inhibitory mechanism was based on the formation of adducts between the flavonoids and phenylacetaldehyde, suggesting that the glucosylation of flavonoids do not modify the mechanism of inhibiting PhIP formation. These findings contribute to a better understanding of the inhibitory effects of hyperoside and quercitrin from ZML on PhIP formation. Different from the flavonoids, chlorogenic acid did not directly react with phenylacetaldehyde to form adducts, it is possible that the thermal degradation products of chlorogenic acid might be responsible for the trapping of phenylacetaldehyde that needs further investigation.

Supplementary information The online version contains supplementary material available at https://doi.org/10.1007/s00217-020-03676-9.

Acknowledgements This research was funded by National Key R\&D Program of China, Grant No. 2019YFC1606200 and the horizontal project of Shanghai Guohui Environmental Protection Technology Group Co., Ltd., Grant No. CTR2020-00392.

\section{Compliance with ethical standards}

Conflicts of interest The authors declare no conflict of interest.
Compliance with ethics requirements This article does not contain any studies with human or animal subjects.

Open Access This article is licensed under a Creative Commons Attribution 4.0 International License, which permits use, sharing, adaptation, distribution and reproduction in any medium or format, as long as you give appropriate credit to the original author(s) and the source, provide a link to the Creative Commons licence, and indicate if changes were made. The images or other third party material in this article are included in the article's Creative Commons licence, unless indicated otherwise in a credit line to the material. If material is not included in the article's Creative Commons licence and your intended use is not permitted by statutory regulation or exceeds the permitted use, you will need to obtain permission directly from the copyright holder. To view a copy of this licence, visit http://creativecommons.org/licenses/by/4.0/.

\section{References}

1. Skog K (1993) Cooking procedures and food mutagens: a literature review. Food Chem Toxicol 31(9):655-675

2. Szterk A (2015) Heterocyclic aromatic amines in grilled beef: the influence of free amino acids, nitrogenous bases, nucleosides, protein and glucose on HAAs content. J Food Compos Anal 40:39-46

3. Helmus DS, Thompson CL, Zelenskiy S et al (2013) Red meatderived heterocyclic amines increase risk of colon cancer: a population-based case-control study. Nutr Cancer 65(8):1141-1150

4. Archer CL, Morse P, Jones RF et al (2000) Carcinogenicity of the $\mathrm{N}$-hydroxy derivative of 2-amino-1-methyl-6-phenylimidazo[4,5$b$ ]pyridine, 2-amino-3, 8-dimethyl-imidazo[4,5-f]quinoxaline and 3, 2'-dimethyl-4-aminobiphenyl in the rat. Cancer Lett 155:55-60

5. Skog KI, Johansson MAE, Jägerstad MI (1998) Carcinogenic heterocyclic amines in model systems and cooked foods: a review on formation, occurrence and intake. Food Chem Toxicol 36(9-10):879

6. Takahashi M, Totsuka Y, Masuda M et al (1997) Reduction in formation of 2-amino-1-methyl-6-phenylimidazo[4,5-b]pyridine 
(PhIP)-induced aberrant crypt foci in the rat colon by docosahexaenoic acid (DHA). Carcinogenesis 10:1937-1941

7. Arlt VM, John G, Schmeiser HH et al (2008) Genotoxicity of 3-nitrobenzanthrone and 3-aminobenzanthrone in MutaMouse and lung epithelial cells derived from MutaMouse. Mutagenesis 6:483-490

8. Zhang Y, Luo Z, Shao Z et al (2014) Effects of antioxidants of bamboo leaves and flavonoids on 2-amino-1-methyl6-phenylimidazo[4,5-b]pyridine (PhIP) formation in chemical model systems. J Agric Food Chem 62:4798-4802

9. Zeng M, Wang J, Zhang M et al (2018) Inhibitory effects of Sichuan pepper (Zanthoxylum bungeanum) and sanshoamide extract on heterocyclic amine formation in grilled ground beef patties. Food Chem 239:111-118

10. Gibis M, Weiss J (2012) Antioxidant capacity and inhibitory effect of grape seed and rosemary extract in marinades on the formation of heterocyclic amines in fried beef patties. Food Chem 134:766-774

11. Ren X, Wang W, Bao Y et al (2020) Isorhamnetin and hispidulin from Tamarix ramosissima inhibit 2-amino-1-methyl6-phenylimidazo[4,5- $b]$ pyridine (PhIP) formation by trapping phenylacetaldehyde as a key mechanism. Foods 9:420

12. Zhu Q, Zhang S, Wang M et al (2016) Inhibitory effects of selected dietary flavonoids on the formation of total heterocyclic amines and 2-amino-1-methyl-6-phenylimidazo[4,5- $b$ ]pyridine $(\mathrm{PhIP})$ in roast beef patties and in chemical models. Food Funct 7(2):1057-1066

13. Guo T, Deng Y, Xie H et al (2011) Antinociceptive and antiinflammatory activities of ethyl acetate fraction from Zanthoxylum armatum in mice. Fitoterapia 82(3):347-351

14. Li J, Hui T, Wang F et al (2015) Chinese red pepper (Zanthoxylum bungeanum Maxim.) leaf extract as natural antioxidants in salted silver carp (Hypophthalmichthys molitrix) in dorsal and ventral muscles during processing. Food Control 56:9-17

15. Singh JP, Kaur A, Singh N et al (2016) In vitro antioxidant and antimicrobial properties of jambolan (Syzygium cumini) fruit polyphenols. LWT Food Sci Technol 65:1025-1030

16. Zeng M, Li Y, He Z et al (2016) Discrimination and investigation of inhibitory patterns of flavonoids and phenolic acids on heterocyclic amine formation in chemical model systems by UPLC-MS profiling and chemometrics. Eur Food Res Technol 242:313-319

17. Tsen SY, Ameri F, Smith JS (2006) Effects of rosemary extracts on the reduction of heterocyclic amines in beef patties. J Food Sci 71(8):C469-C473

18. Cheng K, Wong CC, Cho CK et al (2008) Trapping of phenylacetaldehyde as a key mechanism responsible for naringenin's inhibitory activity in mutagenic 2-amino-1-methyl-6-phenylimidazo [4,5-b]pyridine formation. Chem Res Toxicol 21(10):2026-2034

19. Cheng K, Wong CC, Chao J et al (2009) Inhibition of mutagenic PhIP formation by epigallocatechin gallatevia scavenging of phenylacetaldehyde. Mol Nutr Food Res 53(6):716-725

20. Yang H, Ji Z, Wang R et al (2020) Inhibitory effect of selected hydrocolloids on 2-amino-1-methyl-6-phenylimidazo [4,5-b] pyridine (PhIP) formation in chemical models and beef patties. $\mathrm{J}$ Hazard Mater 402:123486

21. Sabally K, Sleno L, Jauffrit J et al (2016) Inhibitory effects of apple peel polyphenol extract on the formation of heterocyclic amines in pan fried beef patties. Meat Sci 117:57-62

22. Khan IA, Liu D, Yao M et al (2019) Inhibitory effect of Chrysanthemum morifolium flower extract on the formation of heterocyclic amines in goat meat patties cooked by various cooking methods and temperatures. Meat Sci 147:70-81

23. Cheng K, Chen F, Wang M (2007) Inhibitory activities of dietary phenolic compounds on heterocyclic amine formation in both chemical model system and beef patties. Mol Nutr Food Res 51(8):969-976

24. Zhou B, Zhao Y, Wang X et al (2018) Unraveling the inhibitory effect of dihydromyricetin on heterocyclic aromatic amines formation. J Sci Food Agr 98:1988-1994

25. Zochling S, Murkovic M, Pfannhauser W (2002) Effects of industrially produced flavours with pro- and antioxidative properties on the formation of the heterocyclic amine PhIP in a model system. J Biochem Biophys Methods 53(1):37-44

26. Oguri A, Suda M, Totsuka Y et al (1998) Inhibitory effects of antioxidants on formation of heterocyclic amines. Mutation Res 402:237-245

27. Murkovic M (2004) Chemistry, formation and occurrence of genotoxic heterocyclic aromatic amines in fried products. Eur J Lipid Sci Tech 106(11):777-785

28. Zamora R, Hidalgo FJ (2016) The triple defensive barrier of phenolic compounds against the lipid oxidation-induced damage in food products. Trends Food Sci Tech 54:165-174

29. Zhu Q, Zheng ZP, Cheng KW et al (2009) Natural polyphenols as direct trapping agents of lipid peroxidation-derived acrolein and 4-hydroxy-trans-2-nonenal. Chem Res Toxicol 22(10):1721-1727

30. Zhang N, Chen Y, Zhao Y et al (2020) Caffeic acid assists microwave heating to inhibit the formation of mutagenic and carcinogenic PhIP. Food Chem 317:126447

31. Zhao Y, Fan D, Zheng ZP et al (2017) 8-C-(E-phenylethenyl) quercetin from onion/beef soup induces autophagic cell death in colon cancer cells through ERK activation. Mol Nutr Food Res 61(2): 1600437

32. Zheng Z, Yan Y, Xia J et al (2016) A phenylacetaldehyde-flavonoid adduct, 8-C-(E-phenylethenyl)-norartocarpetin, exhibits intrinsic apoptosis and MAPK pathways-related anticancer potential on HepG2, SMMC-7721 and QGY-7703. Food Chem 197:1085-1092

Publisher's Note Springer Nature remains neutral with regard to jurisdictional claims in published maps and institutional affiliations. 